\title{
ON THE THEOREM OF THE PRIMITIVE ELEMENT WITH APPLICATIONS TO THE REPRESENTATION THEORY OF ASSOCIATIVE AND LIE ALGEBRAS
}

\author{
LEANDRO CAGLIERO AND FERNANDO SZECHTMAN
}

\begin{abstract}
We describe of all finite dimensional uniserial representations of a commutative associative (resp. abelian Lie) algebra over a perfect (resp. sufficiently large perfect) field. In the Lie case the size of the field depends on the answer to following question, consider and solved in this paper. Let $K / F$ be a finite separable field extension and let $x, y \in K$. When is $F[x, y]=F[\alpha x+\beta y]$ for some non-zero elements $\alpha, \beta \in F$ ?
\end{abstract}

\section{INTRODUCTION}

That the classification of all finite dimensional indecomposable modules over almost any Lie algebra is a hopeless enterprise follows from [Ma] (see [GP] for the 2-dimensional abelian case).

It is more realistic to concentrate on certain indecomposable modules over some types of Lie algebras. In this regard, recall that a module is uniserial if it is non-zero and its submodules are totally ordered by inclusion. In this paper we consider the problem of describing all finite dimensional uniserial representations of an abelian Lie algebra over an arbitrary field.

This is the starting point of a project aiming to systematically investigate the finite dimensional uniserial representations of distinguished classes of Lie algebras. We have recently classified [CS1] all such representations for the family of complex perfect Lie algebras $\mathfrak{s l}(2) \ltimes V(m)$, where $V(m)$ is the irreducible $\mathfrak{s l}(2)$-module of highest weight $m \geq 1$. This classification turned out to rely on certain zeros of the Racah-Wigner $6 j$-symbol. The analogous problem for a family of solvable Lie algebras over an arbitrary field is considered in [CS2].

In this context, our main result is the following.

Theorem 1.1. Let $\mathfrak{g}$ be an abelian Lie algebra over a perfect field $F$ and let $V$ be a finite dimensional uniserial $\mathfrak{g}$-module. Let $\ell$ be the composition length of $V$ and let $W$ be the socle of $V$. Let $N$ be the number of distinct prime factors of $\operatorname{dim}_{F}(W)$ and suppose that $|F|>N-1$. Then there exists $x \in \mathfrak{g}$ such that $V$ is a uniserial $F[x]$-module. In particular, $x$ acts on $V$, relative to some basis, via the companion matrix $C_{f}$ of a power $f=p^{\ell}$ of an irreducible polynomial $p \in F[X]$, and every other element of $\mathfrak{g}$ acts on $V$ via a polynomial on $C_{f}$.

2000 Mathematics Subject Classification. Primary 17B10, 13C05; Secondary 12F10, 12E20.

Key words and phrases. Uniserial module; Lie algebra; associative algebra; primitive element.

The first author was supported in part by CONICET and SECYT-UNC grants.

The second author was supported in part by an NSERC discovery grant. 
The condition that $F$ be a perfect field such that $|F|>N-1$ is essential. A full account of what happens when $F$ is imperfect is given in Note 3.5. The condition $|F|>N-1$ is related to the following:

Question A. Let $K / F$ be a finite separable field extension and let $x, y \in K$. When is $F[x, y]=F[\alpha x+\beta y]$ for some non-zero elements $\alpha, \beta \in F$ ?

The same question with some replaced by all has a long history, which is explored in detail in $\S 2$. For the stated version, we have the following results.

Theorem 1.2. Let $K / F$ be a finite separable field extension, and let $x, y \in K$ have respective degrees $a=m d$ and $b=$ nd over $F$, where $d=\operatorname{gcd}(a, b)$. Let $N$ be the number of distinct prime factors of $d$ not dividing mn. If $|F|>N+1$ then there are non-zero $\alpha, \beta \in F$ such that $F[x, y]=F[\alpha x+\beta y]$.

Theorem 1.3. Let $K / F$ be a finite separable field extension. Let $N$ be the number of distinct prime factors of $[K: F]$. Suppose that $|F|>N-1$. Then, given any $x_{1}, \ldots, x_{n} \in K$ such that $K=F\left[x_{1}, \ldots, x_{n}\right]$, there is an $F$-linear combination $z=\alpha_{1} x_{1}+\cdots+\alpha_{n} x_{n}$ such that all $\alpha_{i} \neq 0$ and $K=F[z]$.

That the conditions involving $|F|$ in Theorems 1.1, 1.2 and 1.3 are exact is shown in Theorem 2.7.

We remark that Theorem 1.1 is a corollary of a closely related result concerning associative algebras, which reads as follows.

Theorem 1.4. Let $F$ be a perfect field and let $A$ be a finite dimensional commutative and associative algebra over $F$. Then the following conditions are equivalent:

(a) $A=F[u]$ for some $u \in A$ whose minimal polynomial over $F$ is an $\ell$-power of an irreducible polynomial in $F[X]$.

(b) The regular module of $A$ is uniserial of length $\ell$.

(c) A has a finite dimensional faithful uniserial representation of length $\ell$.

Moreover, suppose any of the conditions (a)-(c) is satisfied. Then A has a unique irreducible module, up to isomorphism, namely the residue field, say $R(A)$, of $A$. Let $N$ be the number of distinct prime factors of $[R(A): F]$ and suppose that $|F|>N-1$. Then, given any elements $x_{1}, \ldots, x_{n} \in A$ such that $A=F\left[x_{1}, \ldots, x_{n}\right]$, there is an $F$-linear combination $z$ of $x_{1}, \ldots, x_{n}$ such that $A=F[z]$.

Our proof of Theorem 1.4, given in $\S 3$, is fairly subtle, a key ingredient being the existence and uniqueness of the Jordan-Chevalley decomposition of an endomorphism acting on a finite dimensional vector space over a perfect field. Another consequence of Theorem 1.4 is the following characterization of the finite dimensional uniserial modules of a commutative and associative algebra over a perfect field.

Theorem 1.5. Let $F$ be a perfect field and let $A$ be a commutative and associative $F$-algebra. Let $V$ be a finite dimensional uniserial $A$-module of length $\ell$. Then there exists $x \in A$ such that $V$ is a uniserial $F[x]$-module. In particular, $x$ acts on $V$ via the companion matrix $C_{f}$ of a power $f=p^{\ell}$ of an irreducible polynomial $p \in F[X]$, and every element of $A$ acts on $V$ via a polynomial on $C_{f}$.

To place Theorems 1.1, 1.4 and 1.5 in context, we observe that the class of uniserial modules is very important for associative algebras, whereas for Lie algebras this class has been barely considered. We are confident that it is as relevant as in the associative case and thus worthy to be studied in detail. 
There is an extensive literature on uniserial modules and, more generally, on uniserial and serial rings. Recall that a ring $R$ with identity is called uniserial (resp. serial) if both $R_{R}$ and ${ }_{R} R$ are uniserial (resp. direct sum of uniserial) modules. Here all modules are assumed to be unitary.

Serial rings and algebras occur in several contexts. This class includes discrete valuation rings, Nakayama algebras, triangular matrix rings over a skew field and Artinian principal ideal rings (see $[\mathrm{Pu}],[\mathrm{EG}]$ ). In particular, every proper factor ring of a Dedekind domain is serial. Also, serial algebras occur as the group algebras in characteristic $p$ of certain finite groups, including all $p$-solvable groups with cyclic Sylow $p$-subgroups (see $[\mathrm{Sr}]$ ).

Some important results include the following.

(1) It is due to T. Nakayama [Na, Theorem 17] that a finitely generated module over a serial ring is a serial module (i.e., a direct sum of uniserial modules).

(2) Nakayama algebras (those whose right and left projective modules are uniserial) are of finite representation type, i.e., they have only a finite number of indecomposable modules up to isomorphism (see [ARS, Ch. VI, Theorem 2.1]).

(3) D. Eisenbud and P. Griffith [EG] proved that:

(i) For a finite dimensional $F$-algebra, the serial property is stable under a change of the base field, provided that $A_{0}=A / \operatorname{Rad}(A)$ be separable, i.e., $A_{0} \otimes_{F} K$ is semisimple for every field extension $K / F$.

(ii) The composition series of any uniserial module over a serial ring is periodic in a strong sense (see [EG], Theorem 2.3, for a precise statement).

(iii) Any two simple modules of an indecomposable uniserial ring have the same endomorphism ring.

(4) A complete description of finite dimensional serial algebras over a perfect field is contained in $[\mathrm{AF}]$ and $[\mathrm{DK}]$.

(5) T. Shores and W. Lewis [SL] show that if $M$ is a faithful uniserial module over an integral domain $R$, then $\operatorname{End}_{R}(M)$ is a valuation ring.

\section{Finding PRIMitive ELEMENTS}

Let $K / F$ be an algebraic separable field extension and let $x, y \in K$.

QUESTION 1. When is $F[x, y]=F[\alpha x+\beta y]$ for all non-zero elements $\alpha, \beta \in F$ ?

Question 2. When is $F[x, y]=F[\alpha x+\beta y]$ for some non-zero elements $\alpha, \beta \in F$ ?

Question 1 has been studied by many authors and a brief summary is given below. However, for our purposes, Question 1 only plays a subsidiary role to Question 2, which is an indispensable tool in understanding the finite dimensional uniserial representations of abelian Lie algebras.

By the standard proof of the theorem of the primitive element (see [Ar], Theorem 26) we may restrict our consideration of Question 2 to the case when $F$ is a finite field. In this case, as is well-known, every finite extension of $F$ has a primitive element, but it may surprise the reader to learn that one can always find algebraic elements $x, y$ such that $F[x, y] \neq F[\alpha x+\beta y]$ for all $\alpha, \beta \in F$. Examples are not so easy to construct. It is also unclear at first how to find the exact conditions under which the existence of $\alpha, \beta \in F^{\times}$such that $F[x, y]=F[\alpha x+\beta y]$ is guaranteed. When these conditions fail, our general description of the finite dimensional uniserial representations of abelian Lie algebras over $F$ ceases to be true. 
Our answer to Question 2 is given in Theorems 2.5 and 2.6, while Theorem 2.7 shows that the conditions are exact. Our main applications of the results of this section to representation theory are given in $\S 3$.

Question 1 seems to have been first considered by Nagell [N1], [N2], followed by Kaplansky $[\mathrm{K}]$ and Isaacs $[\mathrm{I}]$. Let $x$ and $y$ have degrees $m$ and $n$ over $F$, respectively. Isaacs showed that if $\operatorname{gcd}(m, n)=1$ then $F[x, y]=F[\alpha x+\beta y]$ for all $\alpha, \beta \in F^{\times}$, provided certain technical conditions hold when $F$ has prime characteristic $p$. The same conclusion was later obtained by Browkin, Diviš and Schinzel [BDS], provided $[F[x, y]: F]=m n$ and different technical condition hold in the prime characteristic case.

Recently, Weintraub [W] showed that if $F[x] / F$ and $F[y] / F$ are Galois extensions, $F[x] \cap F[y]=F$ and $p \nmid \operatorname{gcd}(m, n)$ when $F$ has prime characteristic $p$, then $F[x, y]=F[\alpha x+\beta y]$ for all $\alpha, \beta \in F^{\times}$. We also obtained and proved this result (see Theorem 2.1), unaware at the moment of Weintraub's paper. The condition $p \nmid \operatorname{gcd}(m, n)$ is essential, as seen in Example 2.3. In particular, if $F[x, y] / F$ is an abelian Galois extension and $\operatorname{gcd}(m, n)=1$ then $F[x, y]=F[\alpha x+\beta y]$ for all $\alpha, \beta \in F^{\times}$(see Corollary 2.2). In this regard, see $[\mathrm{P}], \S 3$.

When $F[x] \cap F[y] \neq F$ an answer to Question 1 is more difficult. Sufficient conditions are given in Theorem 2.4, which provides enough information for us to answer Question 2 in Theorems 2.5 and 2.6 when $F$ is a finite field.

Theorem 2.1. Let $K / F$ be an algebraic extension. Let $x$ and $y$ be elements of $K$ of respective degrees $m$ and $n$ over $F$, and satisfying:

(C1) $F[x] / F$ and $F[y] / F$ are Galois extensions.

(C2) $F[x] \cap F[y]=F$.

(C3) If $F$ has prime characteristic $p$ then $p \nmid \operatorname{gcd}(m, n)$.

Then $F[x, y]=F[\alpha x+\beta y]$ for all $\alpha, \beta \in F$ different from 0 .

Proof. It suffices to prove the result for $\alpha=\beta=1$. It is well-known ([DF], $\S 14.4$, Corollary 22) that $F[x, y] / F$ is a Galois extension of degree $m n$.

For $z \in K$ let $S_{z}$ be the stabilizer of $z$ in $\operatorname{Gal}(K / F)$. We need to show that $S_{x+y}$ is trivial. Since $x, y$ generate $F[x, y]$ over $F$, this is equivalent to $S_{x+y} \subseteq S_{x} \cap S_{y}$.

Let $\sigma \in S_{x+y}$. Then

$$
\sigma(x+y)=x+y
$$

SO

$$
\sigma(x)-x=-(\sigma(y)-y)
$$

Since $F[x] / F$ and $F[y] / F$ are normal, this common element, say $f$, belongs to $F[x] \cap F[y]=F$.

Suppose first $\operatorname{char}(F)=0$. Then $\sigma(x)=x+f$ implies $f=0$, since $\sigma$ has finite order. It follows that $\sigma \in S_{x} \cap S_{y}$.

Suppose next $F$ has prime characteristic $p$. Without loss of generality we may assume that $p \nmid m$. From

$$
\sigma(x)=x+f
$$

we infer

$$
\sigma^{p}(x)=x,
$$

so $\sigma^{p} \in S_{x}$. Now $G_{x}$ is normal in $G$ and $G / G_{x}$ has order $m$, so $\sigma^{m} \in G_{x}$. Since $\operatorname{gcd}(p, m)=1$, we deduce $\sigma \in S_{x}$, which implies $f=0$ and a fortiori $\sigma \in S_{y}$, as required. 
Corollary 2.2. Let $K / F$ be a finite abelian Galois extension. If $x, y \in K$ have degrees $m, n$ over $F$ and $\operatorname{gcd}(m, n)=1$ then $F[x, y]=F[\alpha x+\beta y]$ for all $\alpha, \beta \in F^{\times}$.

The condition that $G=\operatorname{Gal}(K / F)$ be abelian in Corollary 2.2 is placed to ensure that every subgroup of $G$ be normal. The groups for which this condition holds are called Dedekind groups. Every Dedekind group is the direct product of the quaternion group $Q_{8}$ with an abelian group [R], so not much is gained by relaxing our hypothesis so that $G$ be a Dedekind group.

Condition (C3) of Theorem 2.1 is redundant if $F$ is a finite field or, more generally, if the Galois group of $F[x, y] / F$ is cyclic. Indeed, in this case

$$
[F[x] \cap F[y]: F]=\operatorname{gcd}(m, n),
$$

so $F[x] \cap F[y]=F$ if and only if $\operatorname{gcd}(m, n)=1$.

However, (C3) cannot be dropped entirely, as the following example shows. This example also illustrates the fact that the element (2.1) of $F$ need not be zero if (C3) fails.

Example 2.3. Given a prime $p$, let $E=F_{p^{2}}$. Let $X, Z$ be algebraically independent over $E$ and let $q=Z^{p^{2}}-Z-X \in E[X, Z]$. It is not difficult to see that $q$ is irreducible in $E[Z][X]$, hence in $E[X, Z]$ and therefore in $E[X][Z]$. Let $F=E(X)$. It follows from Gauss' Lemma that $q \in F[Z]$ is irreducible. Let $\alpha$ be a root of $q$ in some extension of $F$ and set $K=F[\alpha]$. Then $\alpha+a \in K$ is also a root of $q$ for every $a \in E$, so $K / F$ is a Galois extension of degree $p^{2}$. Let $G=\operatorname{Gal}(K / F)$. For each $a \in E$ let $\sigma_{a} \in G$ be given by $\alpha \mapsto \alpha+a$. Then $a \mapsto \sigma_{a}$ is a group isomorphism from the underlying additive group of $E$ onto $G$. In particular, $G \cong C_{p} \times C_{p}$.

Let

Then

$$
x=\alpha(\alpha+1) \times \cdots \times(\alpha+(p-1)) .
$$

Since $\alpha$ has degree $p^{2}$ over $F$, it follows that $x$ is not in $F$. On the other hand,

$$
x^{p}+x-X=\alpha^{p^{2}}-\alpha^{p}+\alpha^{p}-\alpha-X=\alpha+X-\alpha-X=0 .
$$

Thus $x$ is a root of $Z^{p}+Z-X \in F[Z]$. Since $[F[x]: F]>1$ and $[K: F]=p^{2}$, it follows that $[F[x]: F]=p$.

Since $G$ is abelian, $F[x] / F$ is a Galois extension of degree $p$. Here $F[x]$ is the fixed field of $\sigma \in G$, given by $\alpha \mapsto \alpha+1$. In fact,

$$
x=\alpha \times \alpha^{\sigma} \times \cdots \times \alpha^{\sigma^{p-1}} .
$$

Next take $a$ in $E$ but not in $F_{p}$, and let

$$
y=\alpha(\alpha+a) \times \cdots \times(\alpha+(p-1) a) .
$$

Then for $\beta=\alpha / a$, we have

$$
y=a^{p} y / a^{p}=a^{p} \beta(\beta+1) \times \cdots(\beta+(p-1))=a^{p}\left(\beta^{p}-\beta\right)=\alpha^{p}-a^{p-1} \alpha .
$$

In particular, $y$ is not in $F$. On the other hand,

$$
y^{p}+a^{1-p} y-X=\alpha+X-a^{1-p} \alpha^{p}+a^{1-p} \alpha^{p}-\alpha-X=0 .
$$

Thus, $y$ has minimal polynomial $Z^{p}+a^{1-p} Z-X$ over $F$, the extension $F[y] / F$ is Galois of degree $p$, and $F[y]$ is the fixed field of $\tau \in G$, given by $\alpha \mapsto \alpha+a$, with

$$
y=\alpha \times \alpha^{\tau} \times \cdots \times \alpha^{\tau^{p-1}} .
$$


Note that $\sigma$ and $\tau$ both have order $p$ but, by the choice of $a,\langle\sigma\rangle \cap\langle\tau\rangle$ is trivial. In particular, $F[x]$ and $F[y]$ are different subfields of $K$. Degree considerations imply that $F[x] \cap F[y]=F$.

We wish to find all $b, c \in F$ different from 0 such that $K=F[b x+y c]$. Clearly this is equivalent to finding all $b \in F, b \neq 0$, such that $K=F[x+b y]$, and we claim that this holds if and only if $b \notin F_{p} \cdot a$. Thus, all $x+f a y$, where $f \in F_{p}$ is non-zero, have degree $p$ over $F$, while all $x+b y$, where $b \in F$ is not an $F_{p^{-}}$scalar multiple of $a$, have degree $p^{2}$ over $F$.

To see the claim, note that first of all that $G$ consists of all $\sigma^{i} \tau^{j}$, where $i, j \in F_{p}$. Now (2.2) gives

$$
x^{\sigma^{i} \tau^{j}}=(\alpha+j a)^{p}-(\alpha+j a)=x+j\left(a^{p}-a\right),
$$

while (2.3) yields

$$
(b y)^{\sigma^{i} \tau^{j}}=b\left[(\alpha+i)^{p}-a^{p-1}(\alpha+i)\right]=b\left[y+i\left(1-a^{p-1}\right)\right]=b y+i b\left(1-a^{p-1}\right) .
$$

Therefore

$$
(x+b y)^{\sigma^{i} \tau^{j}}=x+b y+j a\left(a^{p-1}-1\right)+i b\left(1-a^{p-1}\right) .
$$

By the choice of $a$, we have $a^{p-1} \neq 1$. Therefore $\sigma^{i} \tau^{j}$ fixes $x+b y$ if and only if $j a=i b$. Clearly $i, j$ are both zero or both non-zero. In the first case we deal with the identity element of $G$, which fixes every element of $K$. In the second case $\sigma^{i} \tau^{j}$ fixes $x+b y$ if and only if $b=j / i \times a$. All in all, $x+b y$ has non-trivial stabilizer in $G$ if and only if $b \in F_{p} \cdot a$, as claimed.

Further information about generalized Artin-Schreier polynomials of the form $Z^{p^{n}}-Z-a$ and their associated Galois extensions, including the computation of primitive elements for every intermediate field, can be found in [GS].

Theorem 2.4. Let $K / F$ be a field extension, let $x, y \in K$ of degrees $a=m d$ and $b=m d$, respectively, with $d=\operatorname{gcd}(a, b)$, and let $E=F[x] \cap F[y]$. Given $\alpha, \beta \in F^{\times}$ let $z=\alpha x+\beta y$ and let $p_{z, E}$ be the minimal polynomial of $z$ over $E$. Assume that:

(i) $F[x] / F$ and $F[y] / F$ are finite Galois extensions.

(ii) $K=F[x, y]$ has degree $m n d=\operatorname{lcm}(a, b)$ over $F$.

Then the degree of $z$ over $F$ is mne, with $e=d /|S|$ and $S$ is the stabilizer of $p_{z, E}$ in $\operatorname{Gal}(E / F)$. Moreover, if $K / F$ is cyclic and $p \mid m n$ for every factor $p$ of $d$ then $K=F[z]$. In particular, if $K / F$ is cyclic and $d \mid m n$ then $K=F[z]$.

Proof. That $K / F$ are $E / F$ are Galois extensions follows from [DF], §14.4, Proposition 21. Here $[E: F]=d$, by $[\mathrm{DF}], \S 14.4$, Corollary 20.

Now $K / E$ is a Galois extension ([DF], $\S 14.2$, Theorem 14) of degree $m n$, where $\operatorname{gcd}(m, n)=1$. Moreover, $E[x]=F[x]$ has degree $m$ over $E$ and $E[y]=F[y]$ has degree $n$ over $E$. It follows from Theorem 2.1 that $K=E[z]$. Thus the degree $z$ over $E$ is $m n$. If $T$ is a transversal for $S$ in $\operatorname{Gal}(E / F)$ then

$$
\prod_{\sigma \in T} p_{z, E}^{\sigma}
$$

is the minimal polynomial of $z$ over $F$ and therefore the degree of $z$ over $F$ is $m n|\operatorname{Gal}(E / F)| /|S|=m n d /|S|$.

Suppose next $\operatorname{Gal}(K / F)=\langle\sigma\rangle$ is cyclic. We claim that if $p$ is a prime and $p \mid m n$ then $\sigma^{m n d / p}(z) \neq z$. Indeed, if $p \mid m$ then $\sigma^{m n d / p}(y)=\sigma^{b m / p}(y)=y$ and, since $\operatorname{gcd}(m, n)=1$, we infer $p \nmid n$, so $\sigma^{m n d / p}(x)=\sigma^{a n / p}(x) \neq x$, whence $\sigma^{m n d / p}(z) \neq z$. 
The case $p \mid n$ is analogous. This proves the claim. Consequently, if every prime factor $p$ of $d$ divides $m n$ then necessarily, $S=1$, so $K=F[z]$.

Theorem 2.5. Let $K / F$ be a finite separable field extension, and let $x, y \in K$ have respective degrees $a=m d$ and $b=n d$ over $F$, where $d=\operatorname{gcd}(a, b)$. Let $A$ be the number of distinct prime factors of $d$ not dividing $m n$. If $|F|>A+1$ then there is $0 \neq \alpha \in F$ such that $F[x, y]=F[x+\alpha y]$.

Proof. By the standard proof of the theorem of the primitive element (see $[\mathrm{Ar}]$, Theorem 26) we may assume that $F$ is finite.

Let $K=F[x, y]$. Since $K / F$ is cyclic, we see that $[F[x] \cap F[y]: F]=d$ and $[K: F]=\operatorname{lcm}(a, b)$.

By Theorem 2.4, if $\alpha \in F, \alpha \neq 0$, then $x+\alpha y$ has degree $m n e_{\alpha}$ over $F$ for some $e_{\alpha} \mid d$. We wish to select $\alpha$ so that $e_{\alpha}=d$.

We have $\operatorname{Gal}(K / F)=\langle\sigma\rangle$, where $\sigma$ has order $m n d$. Suppose $p$ is a prime factor of $d$. If $p \mid m n$ then $\sigma^{m n d / p}(x+\alpha y) \neq x+\alpha y$, while if $p \nmid m n$ then $\sigma^{m n d / p}(x) \neq x$ and $\sigma^{m n d / p}(y) \neq y$, as seen in the proof of Theorem 2.4. In the latter case there is at most one $\alpha \neq 0$ in $F$ such that $\sigma^{m n d / p}(x+\alpha y)=x+\alpha y$.

If $|F|-1>A$ then there is $\alpha \neq 0$ in $F$ such that $x+\alpha y \in$ is not fixed by $\sigma^{m n d / p}$ for any prime factor $p$ of $d$ such that $d \nmid m n$, so $e_{\alpha}=d$ in this case.

Theorem 2.6. Let $K / F$ be a finite separable field extension. Let $B$ be the number of distinct prime factors of $[K: F]$. Suppose that $|F|>B-1$. Then, given any $x_{1}, \ldots, x_{\ell} \in K$ such that $K=F\left[x_{1}, \ldots, x_{\ell}\right]$, there is an $F$-linear combination $z=\alpha_{1} x_{1}+\cdots+\alpha_{\ell} x_{\ell}$ such that all $\alpha_{i} \neq 0$ and $K=F[z]$.

Proof. By the standard proof of the theorem of the primitive element (see [Ar], Theorem 26) we may assume that $F$ is finite. In this case, we argue by induction on $\ell$. If $\ell=1$ there is nothing to do.

Suppose $\ell=2$ and let $p_{1}, \ldots, p_{B}$ be the distinct prime factors of $[K: F]$. Let $a=p_{1}^{a_{1}} \cdots p_{B}^{a_{B}}$ and $b=p_{1}^{b_{1}} \cdots p_{s}^{b_{s}}$ be the respective degrees of $x_{1}$ and $x_{2}$ over $F$, where $a_{i}, b_{j} \geq 0$. If all $a_{i} \leq b_{i}$ we take $z=x_{2}$ and if all $b_{j} \leq a_{j}$ we take $z=x_{1}$. Suppose next $a_{i}>b_{i}$ and $b_{j}>a_{j}$ for some $1 \leq i \neq j \leq B$. Let $d=\operatorname{gcd}(a, b), m=a / d, n=b / d$, and let $A$ be the number of distinct prime factors of $d$ not dividing $m n$. Since $p_{i} \mid m$ and $p_{j} \mid n$, we have $A \leq B-2$. Therefore $|F|-1>B-2 \geq A$, so $K=F[z]$ for some $F$-linear combination $z=\alpha_{1} x_{1}+\alpha_{2} x_{2}$, $\alpha_{i} \neq 0$, by Theorem 2.5 .

Suppose $\ell>2$ and the result is true for $\ell-1$. Let $C$ be the number of distinct prime factors of $\left[F\left[x_{1}, \ldots, x_{\ell-1}\right]: F\right]$. Then $C \leq B$, so $|F|>C-1$. By inductive assumption there is an $F$-linear combination $u=\beta_{1} x_{1}+\cdots+\beta_{\ell-1} x_{\ell-1}, \beta_{i} \neq 0$, such that $F\left[x_{1}, \ldots, x_{\ell-1}\right]=F[u]$. Therefore $K=F\left[u, x_{\ell}\right]$. By the case $\ell=2$ we can find an $F$-linear combination $z=\gamma_{1} u+\gamma_{2} x_{\ell}, \gamma_{i} \neq 0$, such that $K=F[z]$. Since $z=\alpha_{1} x_{1}+\cdots+\alpha_{\ell} x_{\ell}$, with all $0 \neq \alpha_{i} \in F$, the proof is complete.

Theorem 2.7. Given a prime power $q$ and $A \geq q-1$, there exist $a, b \geq 1$ and $x, y$ in some extension of $F=F_{q}$ such that: $d=\operatorname{gcd}(a, b)$ has exactly $A$ distinct prime factors which are not factors of $a / d \times b / d ; x$ has degree a over $F$; $y$ has degree $b$ over $F$; no $F$-linear combination of $x, y$ generates $F[x, y]$ over $F$.

Proof. All degrees considered below are taken over $F=F_{q}$. Let $\left(p_{i}\right)_{i \in F} \times$ be a family of distinct primes in $\mathbb{N}$, and let $r, s$ be primes in $\mathbb{N}$ distinct from each other 
and all $p_{i}$. Let $d$ be the product of all $p_{i}$ and let $t=d r s$. Let $K$ be an extension of $F$ of degree $t$. For $i \in F^{\times}$, let $u_{i} \in K$ be an element of degree $p_{i}$. We further take $x_{0}, y_{0} \in K$ of degree $r$ and $s$, respectively. Let $x$ be the sum of all $i u_{i}$ and $x_{0}$, and let $y$ be the sum of all $u_{i}$ and $y_{0}$. It follows from Corollary 2.2 that $x, y$ have respective degrees $a=t / s$ and $b=t / r$, whence $K=F[x, y]$. Moreover, $\operatorname{gcd}(a, b)=d$. The number of distinct prime factors of $d$ that are not factors of $a / d \times b / d=r s$ is $q-1$. We claim that no $F$-linear combination of $x, y$ generates $K$ over $F$. It suffices to verify this for $x, y$ and all $x-i y, i \in F^{\times}$. Now $s$ does not divide the degree of $x, r$ does not divide the degree of of $y$, and $p_{i}$ does not divide the degree of $x-i y$, as required.

If $A>q-1$, we can easily modify the above construction by selecting $k=$ $A-(q-1)$ further primes, say $q_{1}, \ldots, q_{k}$, multiplying the previous choices of $d$ and $t$ by $q_{1} \cdots q_{k}$, selecting $v_{1}, \ldots, v_{k} \in K$ of respective degrees $q_{1}, \ldots, q_{k}$, and adding the sum of the $v_{i}$ to the previous choices of $x$ and $y$. The same conclusion follows.

\section{Uniserial REPRESENTATIONS OF ABELIAN ASSOCIATIVE AND LiE ALGEBRAS}

Theorem 3.1. Let $F$ be a perfect field and let $A$ be a finite dimensional commutative and associative algebra over $F$. Then the following conditions are equivalent:

(a) $A=F[u]$ for some $u \in A$ whose minimal polynomial over $F$ is an $\ell$-power of an irreducible polynomial in $F[X]$.

(b) The regular module of $A$ is uniserial of length $\ell$.

(c) A has a finite dimensional faithful uniserial representation of length $\ell$.

Moreover, suppose any of the conditions (a)-(c) is satisfied. Then A has a unique irreducible module, up to isomorphism, namely the residue field, say $R(A)$, of $A$. Let $N$ be the number of distinct prime factors of $[R(A): F]$ and suppose that $|F|>N-1$. Then, given any elements $x_{1}, \ldots, x_{n} \in A$ such that $A=F\left[x_{1}, \ldots, x_{n}\right]$, there is an F-linear combination $z$ of $x_{1}, \ldots, x_{n}$ such that $A=F[z]$.

Proof. It is obvious that (a) implies (b) and that (b) implies (c).

Suppose (c) holds. Then there is a finite dimensional $F$-vector space $V$ such that $A$ is a subalgebra of $\operatorname{End}(V)$ and $V$ is a uniserial $A$-module. We wish to show that there is $u \in A$ such that $A=F[u]$, where the minimal polynomial of $u$ is an $\ell$-power of an irreducible polynomial in $F[X]$.

We argue by induction on the composition length $\ell$ of $V$ as an $A$-module. Suppose first that $\ell=1$, that is, $V$ is irreducible. It follows from Schur's Lemma that $D=$ $\operatorname{End}_{A}(V)$ is a finite dimensional division algebra over $F$. Since $A$ is commutative, every $v \mapsto a v, a \in A$, is in $D$. Thus the image of $A$ in $D$ is a finite field extension of $F$, and hence so is $A$ since $V$ is faithful. Since $F$ is perfect, there is $u \in A$ such that $A=F[u]$ (see [Ar], Theorem 27). Clearly, the minimal polynomial of $u$ is irreducible in $F[X]$.

Suppose next that $\ell>1$ and the result is true for faithful uniserial modules of length $<\ell$.

Let $a \in A$. Since $A$ is commutative and $V$ is uniserial over $A$, the minimal polynomial of $a$ must be a power of monic irreducible polynomial $p_{a} \in F[X]$. Moreover, the minimal polynomial of $a$ acting on any finite dimensional irreducible $A$-module must be irreducible. We deduce that the minimal polynomial of $a$ acting on any composition factor of $V$ is $p_{a}$. 
Let $W$ be the socle of $V$. Since $V$ is uniserial, $W$ is irreducible. Let $B$ the subalgebra of $\operatorname{End}(W)$ consisting of all $\left.a\right|_{W}, a \in A$. By above, $B=F\left[\left.b\right|_{W}\right]$ for some $b \in A$. Since $W$ is irreducible as a module for $F[b]$, we see that $\operatorname{dim}(W)=\operatorname{deg}\left(p_{b}\right)$.

Let $C$ be the subalgebra of $\operatorname{End}(V / W)$ of all $\widetilde{a}$, where $a \in A$ and $\widetilde{a}(v+W)=$ $a(v)+W$ for $v \in V$. Note that $V / W$ is a faithful uniserial $C$-module of length $\ell-1$. By inductive hypothesis, there is $x \in A$ such that $C=F[\widetilde{x}]$, where the minimal polynomial of $x$ in $V / W$ is $p_{x}^{\ell-1}$.

We claim that $\operatorname{deg}\left(p_{x}\right)=\operatorname{deg}\left(p_{b}\right)$. Indeed, from $\left.x\right|_{W} \in F\left[\left.b\right|_{W}\right]$ we deduce $\operatorname{deg}\left(p_{x}\right) \leq \operatorname{deg}\left(p_{b}\right)$. Let $U / W$ be the socle of $V / W$, which is an irreducible $A$ module. Let $\hat{x}$ and $\hat{b}$ be the elements of $\operatorname{End}(U / W)$ corresponding to $x$ and $b$, respectively. Since $\hat{b} \in F[\hat{x}]$, we infer $\operatorname{deg}\left(p_{b}\right) \leq \operatorname{deg}\left(p_{x}\right)$, as claimed.

From $\operatorname{deg}\left(p_{x}\right)=\operatorname{deg}\left(p_{b}\right)=\operatorname{dim}(W)$ we deduce that $W$ is an irreducible $F[x]$ module. From now on we let $p=p_{x}$. Thus $x$ has a single elementary divisor $p^{\ell-1}$ on $V / W$ and a single elementary divisor $p$ on $W$. Therefore, one of the following cases must occur:

CASE 1. $x$ has a single elementary divisor $p^{\ell}$. In this case we take $u=x$. Since every $a \in A$ commutes with the cyclic operator $u$, we infer $A=F[u]$.

CASE 2. $x$ has elementary divisors $p^{\ell-1}$ and $p$. Let $R=F[X]$ and view $V$ as an $R$-module via $x$. Then $V=R v_{1} \oplus R v_{2}$, where the annihilating ideals of $v_{1}$ and $v_{2}$ are respectively generated by $p$ and $p^{\ell-1}$. Suppose, if possible, that $\ell>2$. Let $a \in A$. Then $a v_{2}=g(x) v_{2}+h(x) v_{1}$ for some $g, h \in R$. Therefore

$$
a p^{\ell-2}(x) v_{2}=p^{\ell-2}(x) a v_{2}=g(x) p^{\ell-2}(x) v_{2}+h(x) p^{\ell-2}(x) v_{1}=g(x) p^{\ell-2}(x) v_{2} .
$$

Thus $R p^{\ell-2}(x) v_{2}$ is an irreducible $A$-submodule of $V$, so $W=R p^{\ell-2}(x) v_{2}$. But then the minimal polynomial of $x$ acting on $V / W$ is $p^{\ell-2}$, a contradiction. This proves that $\ell=2$.

Thus $V$ is a completely reducible $R$-module, so $V=W \oplus U$, for some irreducible $R$-submodule $U$ of $V$.

But $V$ is not a completely reducible $A$-module, so $A$ is not a semisimple algebra. Therefore, $A$ has a non-zero nilpotent element, say $y$. Hence $p_{y}(X)=X$, so $y$ acts trivially on $W$ and $V / W$. It follows that minimal polynomial of $u=x+y$ is $p$ or $p^{2}$. Suppose, if possible, that the first case occurs. Then $u$ is semisimple and has 2 different Jordan-Chevalley decompositions, which is impossible since $F$ is perfect (see [B], Chapter VII, §5). Thus $u$ has minimal polynomial $p^{2}$, so $V$ is a uniserial $F[u]$-module, and a fortiori $A=F[u]$. This proves (a).

Suppose now the equivalent conditions (a)-(c) hold. Since $A$ is local, it is clear that its residue field $R(A)$ is its unique irreducible module. Let $N$ be the number of distinct prime factors of $[R(A): F]$ and suppose that $|F|>N-1$. Let $x_{1}, \ldots, x_{n}$ be any elements of $A$ satisfying $A=F\left[x_{1}, \ldots, x_{n}\right]$. We wish to show the existence of an $F$-linear combination $z$ of $x_{1}, \ldots, x_{n}$ such that $A=F[z]$. We argue by induction on the composition length $\ell$ of $V=A$ as an $A$-module.

If $\ell=1$ then $A=F[u]$ is a finite field extension of $F$. In this case the existence of $z$ follows from Theorem 2.6.

Suppose next $\ell>1$ and the result is true for algebras of satisfying (a)-(c) of length $<\ell$. Arguing as above, since $C$ is a non-zero factor of the local ring $A$, its residue field is $F$-isomorphic to that of $A$. By inductive hypothesis, there is $v$ in the $F$-span of $x_{1}, \ldots, x_{n}$ such that $C=F[\tilde{v}]$. In Case 1 , when $v$ is cyclic, we can take $z=v$. In Case 2 we have $V=W \oplus U$, where $W$ is the socle of the $A$-module $V$ 
and $W, U$ are irreducible $F[v]$-modules upon which $v$ acts with irreducible minimal polynomial $p_{v}$. Suppose, if possible, that every non-zero $x_{1}, \ldots, x_{n}$ acts semisimply on $V$. Since $F$ is perfect, it follows that every element of $A$ acts semisimply on $V$ (see [B], Chapter VII, §5, Corollary to Proposition 16). This contradicts the fact that $A$ has non-zero nilpotent radical. Thus, there is at least one $i$ such that $y=x_{i} \neq 0$ is not semisimple. Since $F$ is perfect, $y$ has a Jordan-Chevalley decomposition in $\operatorname{End}(V)$ (see [B], Chapter VII, §5), say $y=s+m$, where $s, m \in F[y] \subseteq A, s$ is semisimple, $0 \neq m$ is nilpotent, and $[s, m]=0$. By Theorem 2.6 applied to the finite field extension $B=F\left[\left.v\right|_{W}\right]$ of $F$, there is $0 \neq \alpha \in F$ such that $z=v+\alpha y$ satisfies $B=F\left[\left.z\right|_{W}\right]$. Since $m$ acts trivially on $W$, it follows that that $v+\alpha s$ acts irreducibly on $W$. Now $v$ and $\alpha s$ are semisimple and commute. Since $F$ is perfect, it follows, as above, that $v+\alpha s$ is semisimple. By uniqueness of the Jordan-Chevalley decomposition we see, as above, that $v+\alpha s+\alpha m=z$ has minimal polynomial $p_{z}^{2}$, where $\operatorname{deg}\left(p_{z}^{2}\right)=\operatorname{deg}\left(p^{2}\right)=\operatorname{dim}(V)$. Thus $A=F[z]$, where $z=v+\alpha x_{i}$ is an $F$-linear combination of $x_{1}, \ldots, x_{n}$.

Corollary 3.2. Let $F$ be a perfect field and let $A$ be a commutative and associative $F$-algebra. Let $V$ be a finite dimensional uniserial $A$-module of length $\ell$. Then there exists $x \in A$ such that $V$ is a uniserial $F[x]$-module. In particular, $x$ acts on $V$ via the companion matrix $C_{f}$ of a power $f=p^{\ell}$ of an irreducible polynomial $p \in F[X]$, and every element of $A$ acts on $V$ via a polynomial on $C_{f}$.

Corollary 3.3. Let $F$ be a perfect field and let $\mathfrak{g}$ be an abelian Lie algebra over $F$. Let $V$ be a finite dimensional uniserial L-module of length $\ell$. Let $W$ be the socle of $V$. Let $N$ be the number of distinct prime factors of $\operatorname{dim}(W)$ and suppose that $|F|>N-1$. Then there exists $x \in \mathfrak{g}$ such that $V$ is a uniserial $F[x]$-module. In particular, $x$ acts on $V$ via the companion matrix $C_{f}$ of a power $f=p^{\ell}$ of an irreducible polynomial $p \in F[X]$, and other every element of $\mathfrak{g}$ acts on $V$ via a polynomial on $C_{f}$.

Proof. Apply Theorem 3.1 to the subalgebra of $\operatorname{End}(V)$ generated by the image of $\mathfrak{g}$ under the given representation.

Corollary 3.4. Let $F$ be an algebraically closed field and let $\mathcal{A}$ be an abelian associative or Lie algebra over $F$. Let $V$ be a uniserial $\mathcal{A}$-module of finite dimension $m$. Then there exists an $x$ in $\mathcal{A}$ that is represented by a Jordan block $J_{m}(\alpha), \alpha \in F$, relative to a basis of $V$. Moreover, every other element of $\mathcal{A}$ is represented by a polynomial in $J_{m}(\alpha)$ in that basis.

Note 3.5. It is well-known (see [Ar], Theorem 27) that if $F$ is a perfect field then every finite extension $K$ of $F$ has a primitive element, i.e., an element $x \in K$ such that $K=F[x]$.

However, certain imperfect fields share this property and it is conceivable that the implication $(c) \rightarrow(a)$ of Theorem 3.1 remains valid for these fields, which is certainly the case when the given uniserial module is irreducible. The purpose of this note is to show that the implication $(c) \rightarrow(a)$ of Theorem 3.1 actually fails for every imperfect field, once we allow uniserial non-irreducible modules. In short, the condition that $F$ be perfect is essential to Theorem 3.1.

For the remainder of this note $F$ stands for a field of prime characteristic $p$. Then $F^{p}$ is a subfield of $F$ and the degree $\left[F: F^{p}\right]$ is either infinite or a power of $p$. Following Teichmüller [T], we define the degree of imperfection of $F$ to be infinite or 
$m \geq 0$, depending on whether $\left[F: F^{p}\right]$ is infinite or equal to $p^{m}$. Thus $F$ is perfect if and only if it has degree of imperfection 0 . In this case, if $K=F\left(X_{1}, \ldots, X_{m}\right)$, where $X_{1}, \ldots, X_{m}$ are algebraically independent over $F$, then $K$ has degree of imperfection $m$.

It was shown by Steinitz [St] that every finite extension of an imperfect field of degree of imperfection 1 has a primitive element. For a generalization of Steinitz result see $[\mathrm{BM}]$. It is easy to see that every imperfect field whose degree of imperfection is $>1$ has a finite extension with no primitive element. In particular, Theorem 3.1 does not apply to any of these fields.

Suppose $F$ is imperfect. We claim that there is a commutative and associative $F$-algebra $A$ of dimension $2 p$ with a faithful uniserial $A$-module $V$ of dimension $2 p$ and no $x \in A$ such $A=F[x]$. The key underlying factor here is that the uniqueness part of the Jordan-Chevalley decomposition fails over an imperfect field.

To prove the claim observe that, by hypothesis, there exists $a \in F$ such that $a \notin F^{p}$. Therefore (see $[\mathrm{M}], \S 1.4$, Theorem 9) the polynomial $f=X^{p}-a \in F[X]$ is irreducible. Let $C$ be the companion matrix to $f$ and consider the matrices in $D, E \in M_{2 p}(F)$ defined in terms of $p \times p$ blocks as follows:

$$
D=\left(\begin{array}{cc}
C & 0 \\
0 & C
\end{array}\right), E=\left(\begin{array}{cc}
0 & I_{p} \\
0 & 0
\end{array}\right) .
$$

It is clear that the subalgebra $A$ of $M_{2 p}(F)$ generated by $D$ and $E$ is commutative of dimension $2 p$ with $F$-basis $D^{i} E^{j}$, where $0 \leq i<p, 0 \leq j<1$. Moreover, the column space $V=F^{2 p}$ is a faithful uniserial $A$-module of dimension $2 p$. Let $x \in A$. Then there are $\alpha_{i j} \in F$ such that $x$ is the sum of all $\alpha_{i j} D^{i} E^{j}, 0 \leq i<p, 0 \leq j<1$. Since $E^{2}=0$ and $D^{p}=a I_{2 p}$, it follows that $x^{p}$ is the sum of all $\alpha_{i 0}^{p} a^{i} I_{2 p}, 0 \leq i<p$. Thus, $x^{p}=s I_{2 p}$, where $s \in F$, so $x$ is a root of $X^{p}-s \in F[X]$. In particular, $F[x]$ has dimension $\leq p$, so $F[x]$ is a proper subalgebra of $A$. Note, finally, that $B=D+E$ satisfies

$$
B^{p}=(D+E)^{p}=D^{p}+E^{p}=a I_{2 p},
$$

so the minimal polynomial of $B$ is $f$. In particular, $B$ is semisimple and has 2 different Jordan-Chevalley decompositions.

Note 3.6. Let $A$ be a commutative associative algebra over a field $F$ and let $V$ be a finite dimensional faithful uniserial $A$-module. It is not difficult to see that $V=A v$ must be cyclic, where the annihilator of $v$ is trivial. Then $A \cong A v=V$, so $A$ is uniserial as $A$-module. The argument is taken from the proof of [SL], Proposition 2.1. However, the fact that $A$ itself is uniserial in no way implies the results of this section, as shown in Note 3.5.

Note 3.7. The condition $|F|>N-1$ is essential in Theorem 3.1 and Corollary 3.3, as the irreducible module $F[x, y]$ of Theorem 2.7 shows.

Note 3.8. The condition that $V$ be finite dimensional in Theorem 3.1 is essential. Let $F$ be a field and let $K=F(X)$. The regular module of $K$ is irreducible, but there is no $x \in K$ such that $K=F[x]$.

Note 3.9. The use of uniserial modules in Theorem 3.1 and Corollary 3.3 cannot be extended to more general indecomposable modules. Indeed, let $F$ be a field and let $V$ be a finite dimensional indecomposable but not uniserial module for a Lie or associative algebra. Then there is no $x$ in this algebra such that $V$ is an 
indecomposable $F[x]$-module, for in that case $V$ will be a uniserial $F[x]$-module, and hence a uniserial module for the given algebra.

Note 3.10. The theorem of the primitive element for finite field extensions of perfect fields is a special case of Theorem 3.1.

Acknowledgment. We thank A. Herman for helpful discussions about Theorem 2.7 .

\section{REFERENCES}

[AF] F. Anderson and K. Fuller, Rings and categories of modules, Second edition, Graduate Texts in Mathematics, 13, Springer-Verlag, New York, 1992.

[Ar] E. Artin, Galois Theory, Dover, New York, 1998.

[ARS] M. Auslander, I. Reiten and S. Smalø, Representation theory of Artin algebras, Cambridge Studies in Advanced Mathematics, 36, Cambridge University Press, Cambridge, 1995.

[B] N. Bourbaki, Algebra II, Springer-Verlag, Berlin, 1988.

[BDS] J. Browkin, B. Diviš and A. Schinzel, Addition of sequences in general fields, Monatsh. Math. 82 (1976) 261-268.

$[\mathrm{BM}]$ M. F. Becker and S. MacLane, The minimum number of generators for inseparable algebraic extensions, Bull. Amer. Math. Soc. 46 (1940) 182-186.

[CS1] L. Cagliero and F. Szechtman, The classification of uniserial $\mathfrak{s l}(2) \ltimes V(m)$-modules and a new interpretation of the Racah-Wigner 6j-symbol, J. Algebra 386 (2013) 142-175.

[CS2] L. Cagliero and F. Szechtman, Uniserial modules of certain solvable Lie algebras, in preparation.

[DF] D. S. Dummit and R. M. Foote, Abstract algebra, third edition, John Wiley, NJ, 2004.

[DK] Y. Drozd and V. Kirichenko, Finite-dimensional algebras, Springer-Verlag, Berlin, 1994.

[EG] D. Eisenbud and P. Griffith, Serial rings, J. Algebra 17 (1971) 389-400.

[Fa] A. Facchini, Module theory. Endomorphism rings and direct sum decompositions in some classes of modules, Progress in Mathematics 167, Birkhäuser, Basel 1998.

[GP] I.M. Gelfand, V.A. Ponomarev, Remarks on the classification of a pair of commuting linear transformations in a finite dimensional vector space, Functional Anal. Appl. 3 (1969) 325326.

[GS] N. Guerszenzvaig and F. Szechtman, Generalized Artin-Schreier polynomials, submitted.

[I] I. M. Isaacs, Degrees of sums in a separable field extension, Proc. Amer. Math. Soc. 25 (1970) 638-641.

[K] I. Kaplansky, Fields and Rings, Univ. of Chicago Press, Chicago, 1969.

[M] P. J. McCarthy, Algebraic extensions of fields, Dover, New York, 1991.

[Ma] I. Makedonskyi, On wild Lie algebras, arXiv:1202.1401v2.

[N1] T. Nagell, Bemerkungen über zusammengesetzte Zahlkörper, Avh. Norske Vid. Akad. Oslo (1937) 1-26.

[N2] T. Nagell, Bestimmung des Grades gewisser relativalgebraischer Zahlen, Monatsh. Math. Phys. 48 (1939) 61-74.

[Na] T. Nakayama, On Frobeniusean algebras II, Ann. of Math. 42 (1941) 1-21.

$[\mathrm{P}]$ B. V. Petrenko, On the sum of two primitive elements of maximal subfields of a finite field, Finite Fields Appl. 9 (2003) 102-116.

[Pu] G. Puninski, Serial rings. Kluwer Academic Publishers, Dordrecht, 2001.

[R] D. J. S. Robinson, A course in the theory of groups, Graduate Texts in Mathematics, Springer-Verlag, New York, 1996.

[SL] T. Shores and W. Lewis, Serial modules and endomorphism rings, Duke Math. J. 41 (1974) 889-909.

[Sr] B. Srinisivan, On the indecomposable representations of a certain class of groups, Proc. London Math. Soc. 10 (1960), 497-513.

[St] E. Steinitz, Algebraische Theorie der Körper, J. Reine Angew. Math. 137 (1910) 167-309.

[T] O. Teichmüller, p-Algebren, Deutsche Mathematik, 1 (1936) 362-388.

[W] S. Weintraub, Observations on primitive, normal, and subnormal elements of field extensions, Monatsh. Math. 162 (2011) 239-244. 
Ciem-Conicet, FAMAF-Universidad Nacional de Córdoba, Córdoba, Argentina.

E-mail address: cagliero@famaf.unc.edu.ar

Department of Mathematics and Statistics, University of Regina, Canada

E-mail address: fernando.szechtman@gmail.com 